\title{
EFFECTS OF CHITOSAN/CHONDROITIN SULFATE POLYSACCHARIDE LIDS ON AMOXICILLIN DRUG RELEASE FROM MICROCONTAINERS
}

\author{
1,2llkay KARAKURT, ${ }^{3}$ Khorshid KAMGUYAN, ${ }^{3}$ Carmen Milian GUIMERA, ${ }^{1}$ Kadir OZALTIN, \\ ${ }^{3}$ Anja BOISEN, ${ }^{1,4}$ Marian LEHOCKY \\ ${ }^{1}$ Centre of Polymer Systems, University Institute, Tomas Bata University in Zlin, Zlin, Czech Republic, EU, \\ ykarakurt@utb.cz, ozaltin@utb.cz, lehocky@utb.cz \\ ${ }^{2}$ Department of Health Technology, DTU Health Tech, Technical University of Denmark, Lyngby, Denmark, \\ EU, ilkara@dtu.dk \\ ${ }^{3}$ The Danish National Research Foundation and Villum Foundation's Center for Intelligent Drug Delivery and \\ Sensing Using Microcontainers and Nanomechanics (IDUN), Technical University of Denmark, Lyngby, \\ Denmark,EU, khokam@dtu.dk, aboi@dtu.dk \\ ${ }^{4}$ Faculty of Technology, Tomas Bata University in Zlín, Zlín, Czech Republic, EU, lehocky@utb.cz
}

https://doi.org/10.37904/nanocon.2021.4352

\begin{abstract}
Microcontainers are micrometer-sized cylindrical reservoir-based devices serving as drug carriers for oral administration systems with ensuring a high drug concentration at the desired site of absorption and providing a sustained resident time. These polymeric micro-cylinders consist of drug-filled cavities and can be functionalized by sealing with polymeric lids to protect the active pharmaceutical ingredient against degradation before reaching the absorption site. The present study aimed to develop biopolymer-based lids for microcontainers using polysaccharides for the prolonged release of an antibiotic. Amoxicillin was chosen as a model drug and loaded into microcontainers using the polydimethylsiloxane masking technique, followed by spray coating with chitosan (CS) and chondroitin sulfate (ChS) as polymeric lids onto the cavity of the devices. The structural and physicochemical properties of the microcontainers were investigated. Moreover, the effect of polysaccharide-based lids on the drug release behavior was further analysed and discussed. The thickness and morphology of the lids were characterized by profilometry and SEM before and after spray coating. The in vitro release profiles revealed a fast and an immediate release of amoxicillin from only ChS-coated microcontainers as in the uncoated ones, and slow, prolonged release from only CS-coated ones. However, the microcontainers with CS-ChS lids showed a biphasic release pattern that consists of a lesser burst release in the early stage of treatment, followed by a more sustained release. Therefore, the formation of polyelectrolytes between CS and ChS plays an important role in controlling the release process. The results suggest that the CS-ChS based lids for microcontainers can be useful in biodegradable, tailor-made controlled drug delivery systems.
\end{abstract}

Keywords: Microdevices, polysaccharide lids, spray coating, drug delivery, sustained release

\section{INTRODUCTION}

Bacterial infections have been one of the most prevalent and serious threats to human health for decades [1]. Faced with such a severe situation, controlled use of antibiotics still seems to be the most effective method in infection control [2]. Amoxicillin ( $\alpha$-amino-hydroxybenzylpenicillin), an aminopenicillin antibiotic with broadspectrum antibacterial activity, is used to treat several infections resulted from most gram-positive and gramnegative bacterial pathogens [3]. However, the main limitations associated with this antibiotic are possessing a short half-life and the need for higher doses to effectively show a therapeutic activity which causes severe 
side effects in turn [4,5]. In order to avoid overuse of antibiotics and to improve their pharmacokinetic properties, encapsulation into liposomes, emulsions, polymeric micro-and nanoparticles are being actively developed [6].

Recently, the advances in reservoir-based microdevices such as microcontainers held great promises as oral dosage forms toward delivering the pharmaceuticals to the absorption site, providing a unidirectional release, and improving oral bioavailability [7]. In addition, with the functionalization by polymeric lids, controlled drug release profiles and additional mucoadhesion can be achieved [8].

Among natural polysaccharides, chitosan and chondroitin sulfate have been widely used as release modifiers in drug delivery applications owing to their unique properties, including biodegradability, antibacterial activity, and excellent biocompatibility [9]. Chitosan acts as a polycation due to the free $\mathrm{NH}_{2}$ groups in its structure, which in turn reacts with negatively charged substances such as chondroitin sulfate, that consists of $\mathrm{SO}_{4}{ }^{2-}$ and COO- groups, making it an optimal candidate to form a polyelectrolyte complex (PEC) $[10,11]$.

In this study, a BCS class I antibiotic was loaded into microcontainers through the polydimethylsiloxane (PDMS) masking technique and were coated with the polysaccharides by using the Layer-by-layer (LBL) spraying technique. The microcontainers were functionalized by applying two layers as lids on top of the microcontainers, the first layer was either chitosan or chondroitin sulfate. The amoxicillin loaded and polysaccharide-coated microcontainers were evaluated in vitro for morphology and drug release properties.

\section{MATERIALS AND METHODS}

Silicon (Si) wafers (4-in, b100N n-type) were obtained from Okmetic (Vantaa, Finland), and SU-8 2075 and SU-8 developers were purchased from Microresist Technology GmbH (Berlin, Germany). Low molecular weight chitosan $(50-190 \mathrm{kDa})$ with $75-85 \%$ degree of deacetylation, chondroitin sulfate A sodium salt $(60 \%$ balance is chondroitin sulfate C), acetic acid (100.5\%) and PBS tablets were purchased from Sigma Aldrich (Prague, Czech Republic). Sylgard $B 184$ two-part Silicone Elastomer (viscosity (mixed) $=3500 \mathrm{cP}$, specific gravity $($ cured $)=1.03$ ) was obtained from Dow Corning $(\mathrm{MI}, \mathrm{USA})$. Amoxicillin trihydrate was provided from Tokyo Chemical Industry (Tokyo,Japan) and Milli-Q deionized water was supplied by a MilliQ Integral Water Purification System for Ultrapure Water, produced by Merck Millipore (Burlington, MA, USA).

\subsection{Microcontainers Fabrication}

Microcontainers were produced on silicon wafers using the negative epoxy-based photoresist SU-8 with two steps of photolithography technique [12]. Briefly, SU-8 was dispensed onto Si wafers and spin coated in two steps. To remove the solvent from the resulting SU-8 film, a soft-baked step was involved and followed by selective exposure to UV light through a chromium mask. The polymerization of exposed walls was induced by post-exposure baking. Defining the sidewalls of microcontainers were achieved with a second spin coating of SU-8, UV lithography, and baking steps. After immersion in propylene glycol methyl ether acetate (PGMEA), and rinsing in isopropyl alcohol, microcontainers containing wafers were cut into $1.2 \times 1.2 \mathrm{~cm}$ chips using an automatic dicing saw (DAD 321, DISCO, Tokyo, Japan).

\subsection{Drug Loading}

The microcontainers were manually filled with amoxicillin using the PDMS-masking technique [13] (Figure 1). Briefly, resin and curing agent of Sylgard $® 184$ were mixed $(10: 1 \mathrm{w} / \mathrm{w}$ ratio) and slowly poured in microcontainer-containing wells in the chip tray. After the gaps between microcontainers fully covered by PDMS, the chip tray was put into the oven and kept $30 \mathrm{~min}$ at $70{ }^{\circ} \mathrm{C}$ for curing. Amoxicillin was filled into the microcontainers using a spatula to press onto the powder and excess drug was swept using a soft brush. The PDMS mask was gently peeled off by pulling through the edges and detached from the Si wafer bearing the 
drug-loaded microcontainers. The amount of the drug was calculated by weighing chips before and after loading.
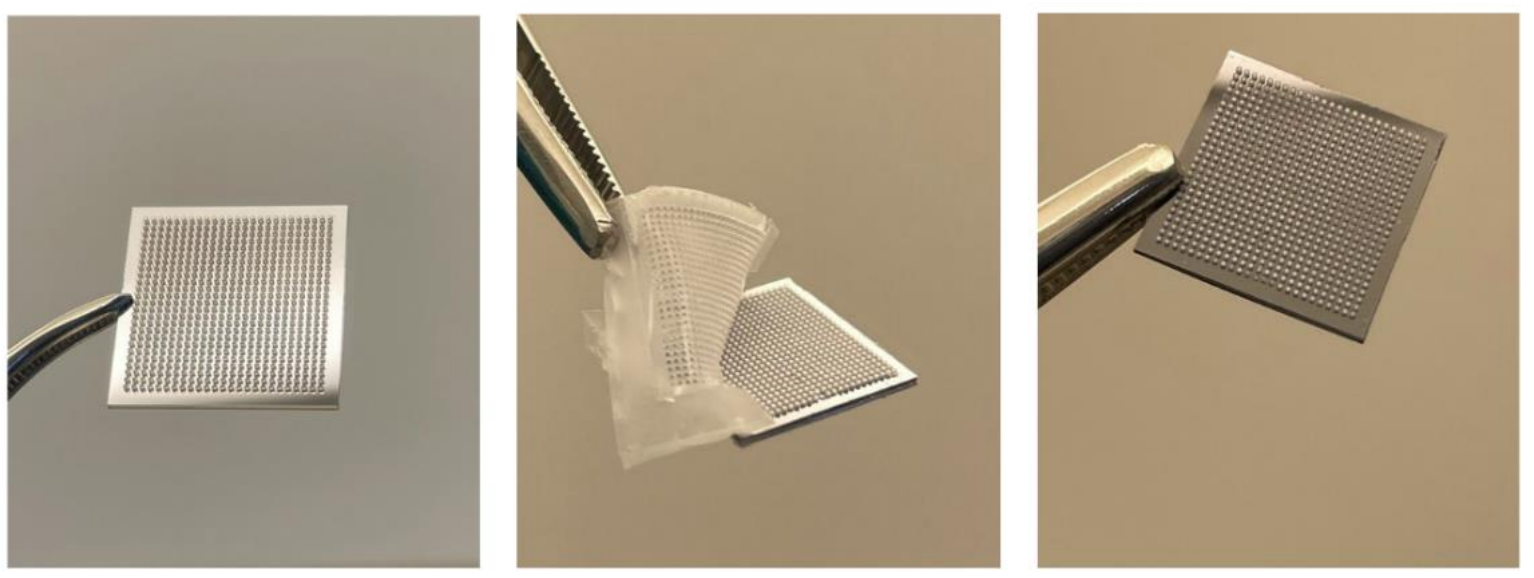

Figure 1 Empty microcontainers on Si chip (a), peeling off PDMS masking after drug loading (b), drugloaded microcontainers (c)

\subsection{Spray Coating}

Amoxicillin-loaded microcontainers were coated with either CS, ChS or their combination by using an ultrasonic spray coater (ExactaCoat, Sono Tek, USA) with an Accumist nozzle. For spray coating, 0.5\% (w/v) concentration of chondroitin sulfate in Milli $Q$ water and $0.5 \%(\mathrm{w} / \mathrm{v})$ chitosan solution in $0.1 \mathrm{M}$ acetic acid were prepared separately. The solutions were sprayed onto the microcontainers with a $1.3 \mathrm{~W}$ generator power and coated in a total of 100 loops.

\subsection{Morphological Characterization}

The microcontainers were characterized before and after loading and coating processes using a TM3030Plus tabletop scanning electron microscope (SEM, Hitachi High Technologies Europe GmbH, Germany) with mounting on a metallic holder with a tilt of $30^{\circ}$ and analyzed at a voltage of $15 \mathrm{keV}$.

The dimensions of the empty microcontainers and the thickness of the coating were measured using optical and contact profilometry using a $3 \mathrm{mg}$ tip force with a scan speed of $100 \mu \mathrm{m} / \mathrm{s}$.

\subsection{Drug Release}

Amoxicillin release from the coated and uncoated microcontainers was measured using a $\mu$-Diss Profiler (Pion Inc. Woburn, MA, USA) with a temperature-controlled water bath at $37^{\circ} \mathrm{C}$. Each chip was attached to a cylindrical magnetic stirrer and placed into a $10 \mathrm{~mL}$ of PBS-filled glass vial. The absorbance was measured at $270 \mathrm{~nm}$. The release profiles were monitored in real-time through in situ UV probes with a path length of $5 \mathrm{~mm}$ and each channel was calibrated with drug concentrations of 0.05 to $0.5 \mathrm{mg} / \mathrm{mL}$. The cumulative release profiles were calculated at each time point and presented as mean \pm SD.

\section{Results}

Two different types of profilometry were used to acquire topographical properties of empty microcontainers and step height measurements of coating. As can be seen from the three-dimensional profiles of the empty microcontainers in Figure 2, they have an inner diameter of $232 \pm 1 \mu \mathrm{m}$ and an inner height of $272 \pm 3 \mu \mathrm{m}$ (mean $\pm S D, n=3$ ). 

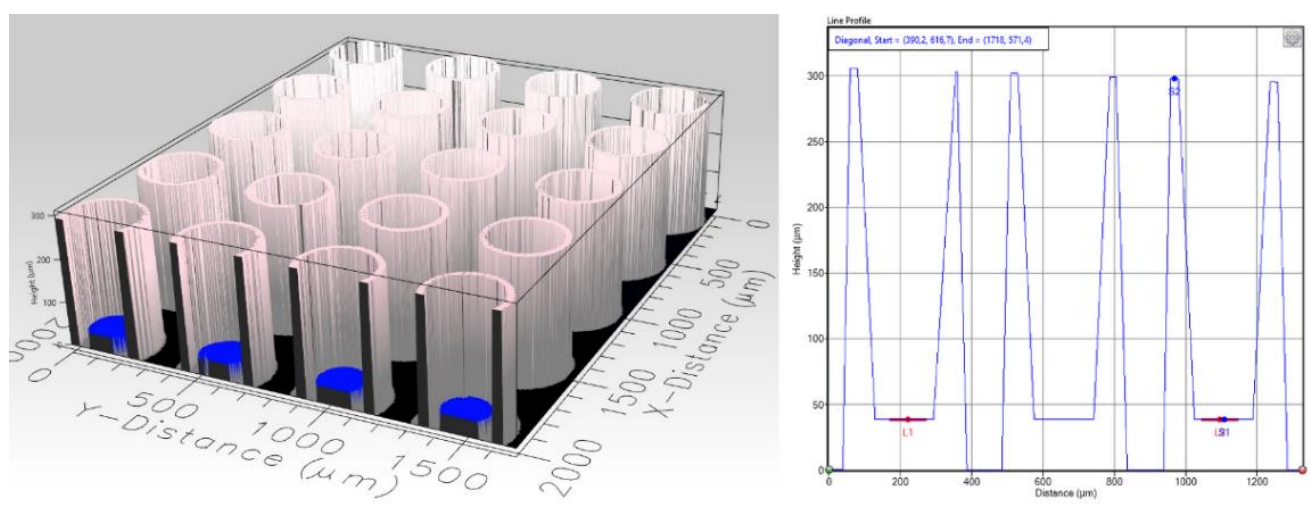

Figure $22 \mathrm{D}$ and 3D profiles of empty microcontainers

The microcontainers were subjected to spray coating of CS or ChS solutions separately and in combination with the parameters described in Table 1. For the layer-by-layer coating, the first layer was either CS or ChS, and the next layer consisted of 50 loops of the counterpart polysaccharide that resulted in a total of 100 loops coating. The thicknesses of the different coatings were measured using a stylus profilometer. The highest lid thickness was obtained with CS due to having a higher density, whereas ChS coating was resulted in a lower value.

Table 1 The parameters of the spray coating and the thicknesses of the different coatings

\begin{tabular}{|c|c|c|c|}
\hline \multirow{2}{*}{ Parameters } & \multicolumn{3}{|c|}{ Coatings } \\
\cline { 2 - 4 } & CS & ChS & CS/ChS \\
\hline Path speed $\left[\mathrm{mm} \mathrm{s}^{-1}\right]$ & 20 & 20 & 20 \\
\hline Infusion rate $[\mathrm{mL} \mathrm{min-1]}$ & 0.05 & 0.04 & $0.05 / 0.04$ \\
\hline Generator power $[\mathrm{W}]$ & 1.3 & 1.3 & 1.3 \\
\hline Shaping air pressure $[\mathrm{kPa}]$ & 0.012 & 0.01 & $0.012 / 0.01$ \\
\hline Heat plate temperature $\left[{ }^{\circ} \mathrm{C}\right]$ & 50 & 35 & $50 / 35$ \\
\hline No. of loops & 100 & 100 & $50 / 50$ \\
\hline Thickness $(\mu \mathrm{m})$ & $6.37 \pm 1.33$ & $4.49 \pm 1.15$ & $5.50 \pm 0.74$ \\
\hline
\end{tabular}
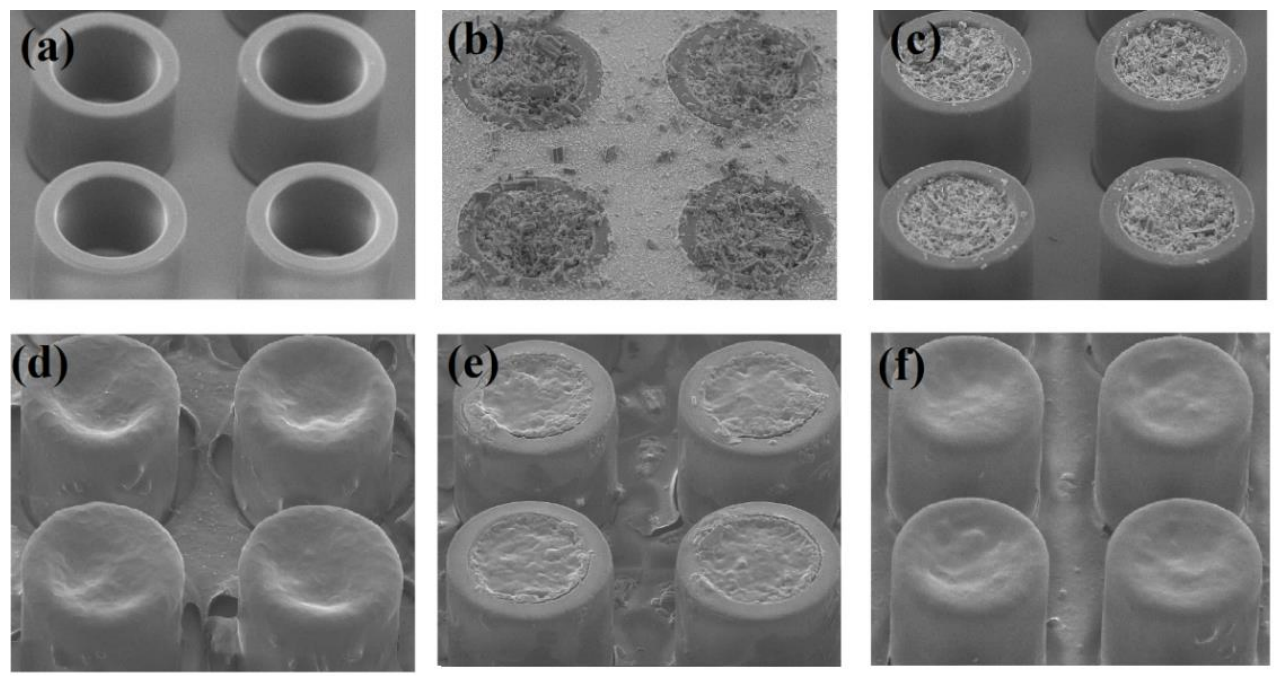

Figure 3 SEM images of empty (a), PDMS-masked drug loaded (c), after peeling of the masking (d), CS (e), $\mathrm{ChS}(\mathrm{f})$ and $\mathrm{CS} / \mathrm{ChS}$ coated microcontainers 
Figure 3 shows SEM images of uncoated, PDMS-masked drug loading and spray coated microcontainers. As can be seen, the drug loadings were efficient, the coatings were homogenous and successfully sealed the drug-loaded microcontainers.

In vitro release profile of uncoated and polysaccharides-coated amoxicillin loaded microcontainers is shown in Figure 4. A cumulative release of $97.3 \pm 10.3 \%$ for uncoated, $89.1 \pm 3.1 \%$ for CS coated and $91.5 \pm 9.2 \%$ for ChS coated microcontainers were obtained in $15 \mathrm{~h}$. Within the first hour, an initial burst release of over $60 \%$ of amoxicillin occurred for both uncoated and ChS containing microcontainers. However, CS ones showed slower release as can be clearly seen in the inset of Figure 4. Nevertheless, CS microcontainers continued their fast release profile due to the hydration and swelling, reached and passed uncoated and ChS coated ones within $2 \mathrm{~h}$. Microcontainers with CS-ChS and ChS-CS as lids, followed significantly slower and sustained release patterns and reached $74.7 \pm 4.8 \%$ and $73.8 \pm 2.9 \%$ after $14 \mathrm{~h}$. This behavior can be attributed to the fact that PEC formation between CS and ChS would cause the lids to be less susceptible to swelling and restrain the release of amoxicillin [14].

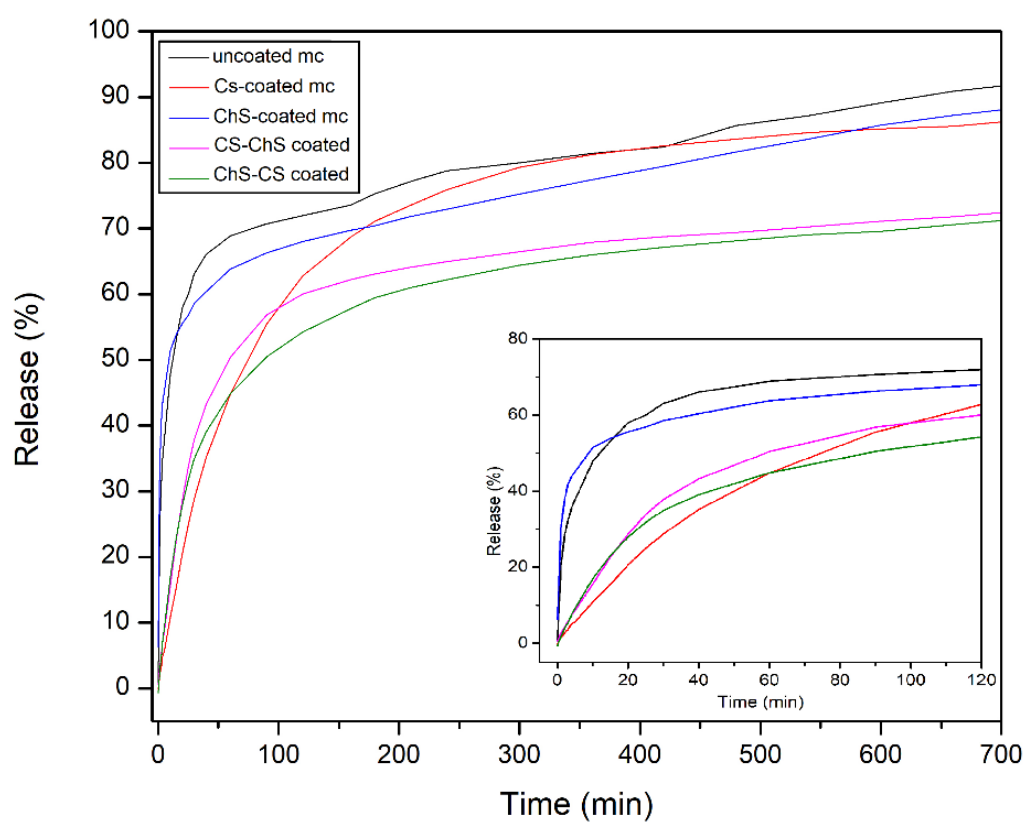

Figure 4 Cumulative release profile of amoxicillin from uncoated and coated microcontainers $(\mathrm{mc})$

\section{CONCLUSION}

In this study, the influence of the combination of polysaccharides as polymeric lids for microcontainers was evaluated for an oral drug delivery system. Following the successful drug loading through the PDMS masking technique, the microcontainers were coated with either CS, ChS or their combination using spray coating. SEM images and thickness measurements with profilometer showed homogenous coatings of microcontainers. The results of in vitro release studies confirm that the microcontainers with CS-ChS lids showed a slower and prolonged release (regardless of the layer order) compared to the individual counterparts, which proves the PEC formation between the two polysaccharides. It is expected that using CS-ChS as release controlling polymer lids could be a promising therapeutic alternative to enhance the oral delivery of antibiotics by increasing the residence time.

\section{ACKNOWLEDGEMENTS}

This research funded by Internal Grant Agency of Tomas Bata University in Zlín, Czech Republic (IGA/CPS/2021/001). 


\section{REFERENCES}

[1] WANG, X., DENG, A., CAO, W., LI, Q., WANG, L., ZHOU, J., HU, B., XING, X. Synthesis of chitosan/poly (ethylene glycol)-modified magnetic nanoparticles for antibiotic delivery and their enhanced anti-biofilm activity in the presence of magnetic field. Journal of Materials Science. 2018, vol. 53, no. 9, pp. 6433-6449.

[2] SMITH, K. J., MOSHREF, A. R., JENNINGS, J. A., COURTNEY, H. S., HAGGARD, W. O. Chitosan sponges for local Synergistic infection Therapy: A pilot study. Clinical Orthopaedics \& Related Research. 2013, vol. 471, no. 10, pp. 3158-3164.

[3] TRUCILlO, P., FERRARI, P., CAMPARDELLI, R., REVERCHON, E., PEREGO, P. A supercritical assisted process for the production of amoxicillin-loaded liposomes for antimicrobial applications. The Journal of Supercritical Fluids. 2020, vol. 163, p. 104842.

[4] LOPES-DE-CAMPOS, D., M. PINTO, R., COSTA LIMA, S. A., SANTOS, T., SARMENTO, B., NUNES, C., REIS, $\mathrm{S}$. Delivering amoxicillin at the infection site- a rational design through lipid nanoparticles. International Journal of Nanomedicine, 2019, vol. 14, pp. 2781-2795.

[5] XU, J., XU, B., SHOU, D., XIA, X., HU, Y. Preparation and evaluation of vancomycin-loaded n-trimethyl chitosan nanoparticles. Polymers. 2015, vol. 7, no. 9, pp. 1850-1870.

[6] NIJLAND, R., HALL, M., BURGESS, J. Dispersal of BIOFILMS BY Secreted, Matrix degrading, bacterial dnase. Biofilm Control and Antimicrobial Agents. 2014, pp. 27-43.

[7] BIRK, S. E., HAAGENSEN, J. A., JOHANSEN, H. K., MOLIN, S., NIELSEN, L. H., BOISEN, A. Microdevices: Microcontainer delivery of Antibiotic improves treatment of Pseudomonas aeruginosa biofilms (adv. Healthcare Mater. 10/2020). Advanced Healthcare Materials. 2020, vol. 9, no. 10, 2070027.

[8] ABRUZZO, A., GIORdANI, B., MITI, A., VITALI, B., ZUCCHERI, G., CERCHIARA, T., BIGUCCI, F. Mucoadhesive and mucopenetrating chitosan nanoparticles for glycopeptide antibiotic administration. International Journal of Pharmaceutics. 2021, vol. 606, 120874.

[9] AMRUTKAR, J. R., GATTANI, S. G. Chitosan-Chondroitin sulfate based Matrix tablets for Colon specific delivery of Indomethacin. AAPS PharmSciTech. 2009, vol. 10, no. 2, pp. 670-677.

[10] SHARMA, S., SWETHA, K. L., ROY, A. Chitosan-Chondroitin sulfate based polyelectrolyte complex for effective management of Chronic wounds. International Journal of Biological Macromolecules. 2019, vol. 132, pp. 97-108.

[11] LAYEK, B., MANDAL, S. Natural polysaccharides for controlled delivery of oral therapeutics: A recent update. Carbohydrate Polymers. 2020, vol. 230, 115617.

[12] NIELSEN, L. H., KELLER, S. S., GORDON, K. C., BOISEN, A., RADES, T., MÜLLERTZ, A. Spatial confinement can lead to increased stability of amorphous indomethacin. European Journal of Pharmaceutics and Biopharmaceutics. 2012, vol. 81, no. 2, pp. 418-425.

[13] KAMGUYAN, K., THAMDRUP, L. H., VAUT, L., NIELSEN, L. H., ZOR, K., BOISEN, A. Development and characterization of a pdms-based masking method for microfabricated oral drug delivery devices. Biomedical Microdevices. 2020, vol. 22, no. 2.

[14] KAUR, G., RANA, V., JAIN, S., TIWARY, A. K. Colon delivery of Budesonide: Evaluation of Chitosan-Chondroitin Sulfate interpolymer complex. AAPS PharmSciTech. 2009, vol. 11, no. 1, pp. 36-45. 